\title{
Application of a local search strategy improves the detection of blood flow deficits in the neuroretinal rim of glaucoma patients using scanning laser Doppler flowmetry
}

\author{
Sarah L Hosking, Sally J Embleton, Ian A Cunliffe
}

\begin{abstract}
Backgroundlaim-Blood flow measures acquired using the scanning laser Doppler flowmeter (SLDF) are known to be highly susceptible to spatial and temporal variations of physiological origin. The purpose of this study was to evaluate a local search strategy intended to overcome these intrinsic variations, thereby improving the detection of blood flow defects resulting from glaucoma.
\end{abstract}

Methods-The sample consisted of one eye of each of 15 glaucoma patients (aged 69.1 (SD 6.6) years) and 15 normal subjects (aged 65.2 (13.7) years). Three 10 degree images of the superior temporal retina and three images of the superior temporal rim were acquired using the Heidelberg retina flowmeter (HRF). Standard analysis was performed using a $10 \times 10$ pixel frame. For the search strategy the same frame was located within a $15 \times$ 15 pixel window and manually repositioned in order to identify the highest and lowest local values of blood flow. Student's paired $t$ test was used to identify differences between groups for the two methods $(\mathbf{p}<0.05)$.

Results-The standard strategy revealed no significant differences in blood flow measures between the subjects at either the retina or neuroretinal rim. With the search strategy there was also no difference in blood flow measures at the retina. At the neuroretinal rim, the search strategy demonstrated that the highest measured blood flow, volume, and velocity values were significantly lower for the glaucoma patients $(p=0.002,0.02$, and 0.002 respectively) while comparison of the lowest flow values showed that glaucoma patients had lower blood flow and velocity only ( $p=0.023$ and 0.021 respectively).

Conclusions-Glaucoma patients exhibit reduced ocular blood flow at the neuroretinal rim, which seems to affect high velocity flow more profoundly than low velocity flow. When analysing perfusion images a local search strategy is recommend to identify the highest local blood flow values in order to optimise the ability to differentiate between subject groups. (Br F Ophthalmol 2001;85:1298-1302)
The scanning laser Doppler flowmeter (SLDF) measures blood flow, volume, and velocity in the retina and neuroretinal rim. It combines the optical Doppler effect with scanning laser flowmetry to give values of capillary blood flow, volume, and velocity measured in arbitrary units. Infrared light with a wavelength of $780 \mathrm{~nm}$ is emitted from the instrument and reflected from both stationary and moving objects. Interference occurs between light reflected from the relatively stationary background, such as the blood vessel walls, and that reflected from moving objects, such as the red blood cells. This interference results in a beat frequency, which is proportional to blood corpuscle velocity. ${ }^{2}$ This beat frequency is detected and measured by a photodiode detector. Following fast Fourier transformation, blood flow measurements are obtained from two dimensional perfusion images. Values of blood flow, volume, and velocity are acquired by placing a $10 \times 10$ pixel frame at the neuroretinal rim or peripapillary retina.

Investigations using the SLDF, in this case, the Heidelberg retina flowmeter (HRF, Heidelberg Engineering, Germany) have shown that it yields reproducible values of retinal perfusion. ${ }^{3-5}$ However, a particular difficulty with this method is that when comparing blood flow values between images for a patient, even when using a carefully selected predetermined location, measurements can vary significantly. Large variations are often noted when the measurement box is moved by as little as 1 or 2 pixels. This is the result of, at least in part, normal physiological variations in blood flow arising from the cardiac cycle, which may influence the actual positioning of the capillary bed or the phase of the pulsation cycle during which data are acquired. The incorporation of pulse synchronisation during data acquisition has been shown to reduce the consequences of the spatial and temporal variability induced by systemic circulatory variation. ${ }^{6}$ The manufacturers recommend that blood flow measures be obtained by choosing a location on the retina or neuroretinal rim and measuring blood flow parameters within a $10 \times 10$ pixel grid. ${ }^{3}$ If the vascular network is not stationary relative to the retina retracing of the same location using a mapping technique can be difficult. This will result in variability in measured flow, which is difficult to account for.

The HRF has been used to investigate blood flow deficits in patients with normal tension 
and primary open angle glaucoma. ${ }^{7-10}$ Significant reductions in blood flow measures have been reported in patients with primary open angle glaucoma in the retina ${ }^{7}$ and lamina cribrosa $^{9}$ and for normal tension glaucoma patients in the peripapillary retina. ${ }^{8}$ At the neuroretinal rim, blood flow measurements have been reported as being lower by some authors ${ }^{7}$; however, this has not been a universal finding. ${ }^{9}$ It is possible that some of these differences in reported findings can be explained by the effects of a local physiological variability, which is not accounted for during the analysis.

The purpose of this study was to determine whether blood flow deficits are more readily detected when a search strategy, involving the lowest or highest local values of flow, is applied when compared. Further, the possibility of a preferential loss in the number or rate of moving corpuscles within a specific range of velocities has not previously been reported.

\section{Methods}

Fifteen glaucoma patients (mean age 69.1 (SD 6.6) years) and 15 age matched normal subjects (mean age 65.2 (13.7) years) were recruited. Glaucoma patients were recruited from outpatient clinics at Birmingham Heartlands Hospital; normal subjects were recruited from spouses of patients or were members of staff from the hospital. Both subject groups were required to have a visual acuity of $6 / 9$ or better in each eye, a refraction of less than 8 dioptres mean sphere, no history of ocular trauma or surgery, and no diabetes mellitus. The normal subjects were required to have no family history of glaucoma. Ocular examination in the age matched normal subjects confirmed open anterior chamber angles and normal optic nerve head morphology. Intraocular pressures in the normal subject group were 13.7 (SD 3.3) mm Hg. Glaucoma patients exhibited repeatable mild to moderate Humphrey 24-2 visual field defects (average mean defect $-5.44(2.3) \mathrm{dB}$ ) as defined by Hodapp et $a l^{11}$ and confirmed optic nerve head cupping consistent with a diagnosis of glaucoma. All glaucoma patients were taking ocular hypotensive medications with mean intraocular pressures of 15.8 (3.8) $\mathrm{mm} \mathrm{Hg}$.

One eye of each of the subjects alternately selected so that approximately equal numbers of right and left eyes were included. Three 10 degree images were taken of the neuroretinal rim, and three of the superotemporal retina, using the HRF. Retinal analysis was undertaken within 1.5 disc diameters of the rim, superior and temporal to the disc, and avoiding larger blood vessels. This area was chosen for image acquisition of the retina and neuroretinal rim as it has been shown to exhibit the least variability in blood flow measures. ${ }^{12}$ Fast Fourier transformation was used to derive perfusion images. Images were included if the (direct current) DC value within the measurement area was between 110 and 150 arbitrary units (AU).

Blood flow, volume, and velocity (arbitrary units) were determined for each image using the $10 \times 10$ pixel square grid located at a

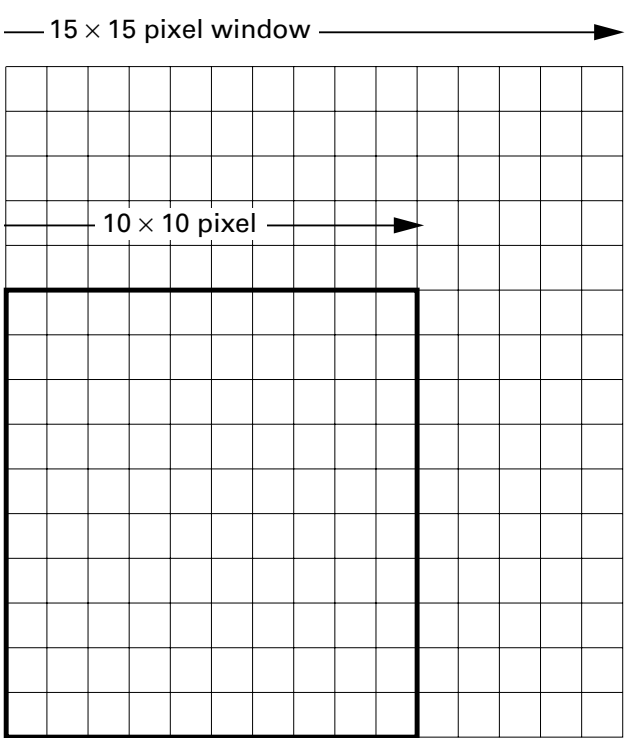

Figure 1 Diagram illustrating the search strategy, showing a $10 \times 10$ pixel frame for analysis within a $15 \times 15$ pixel window.

predetermined position on the retina and rim of each subject. Using the standard method the $10 \times 10$ pixel frame was repositioned at the same location of the retina and neuroretinal rim in each image to obtain blood flow measures. Using acetate sheets the vascular network of the retina and neuroretinal rim was mapped out in each patient and the position of the $10 \times 10$ pixel frame was marked on the sheet to facilitate good repositioning in each image for both areas.

Using the search strategy the $10 \times 10$ pixel frame was systematically repositioned within a $15 \times 15$ pixel window located at a similar position on the retina or neuroretinal rim (Fig 1). The highest and lowest local values of blood flow, volume, and velocity were identified and recorded.

Full ethical approval was granted before the start of the study from the institutions involved and informed consent was obtained from each subject. All procedures conformed to the tenets of the Declaration of Helsinki.

\section{STATISTICAL ANALYSIS}

The mean and standard deviations of blood flow, volume, and velocity were determined for the standard strategy and search strategy (highest and lowest values) for each eye at the retina and neuroretinal rim for the glaucoma patients and the age matched normal subjects. Group mean values and standard deviations were determined for the retina and rim locations for both methods. Student's paired $t$ tests were used to identify significant differences in blood flow, volume, and velocity for the two groups at the retina and neuroretinal rim using both methods.

\section{Results}

At the retina, no significant difference was found between the two groups for blood flow, volume, and velocity ( $\mathrm{p}>0.05)$ using either method. 

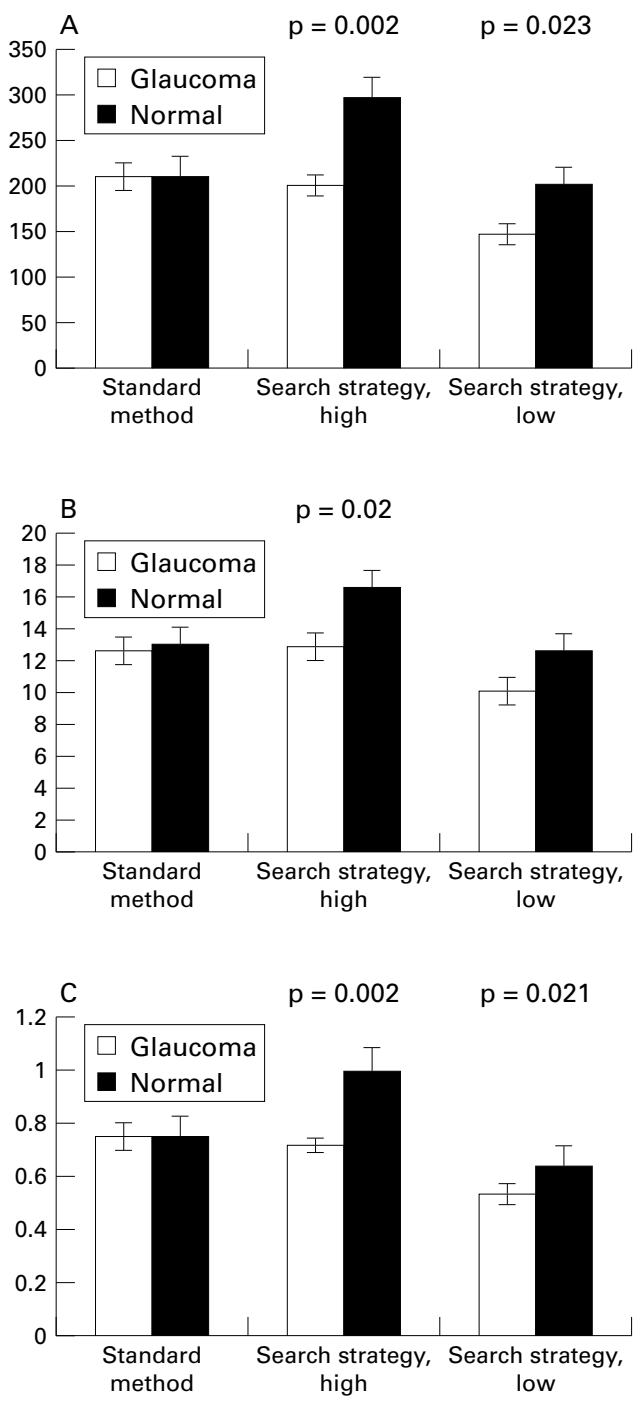

Figure 2 Graphs showing the normal and glaucoma group mean values for ocular blood (A) flow, $(B)$ volume, and $(C)$ velocity (arbitrary units) measured using the standard method and the search method. No differences were found between groups using the standard strategy. Using the search strategy, significant differences between groups were found for the highest values of blood flow ( $p=$ $0.002)$, volume $(p=0.02)$, and velocity $(p=0.002)$ and for the lowest flow $(p=0.023)$ and velocity $(p=0.021)$.

At the neuroretinal rim the standard technique revealed no significant difference between groups for blood flow, volume, or velocity. Using the search strategy, significant perfusion reductions were found in the glaucoma group for the highest measured blood flow, volume, and velocity values $(\mathrm{p}=0.002,0.02$, and 0.002 respectively). When the lowest blood flow, volume, and velocity measures were evaluated the difference was less marked, reaching significance for blood flow and velocity $(\mathrm{p}=0.023$ and $p=0.021$ respectively) but not for blood volume $(p=0.07)$. Figure 2 shows the neuroretinal rim blood flow, volume, and velocity measured using the two strategies for both the glaucoma and normal subject groups.

\section{Discussion}

Using the standard (static) method to acquire measures of blood flow no significant difference was found between the two groups at

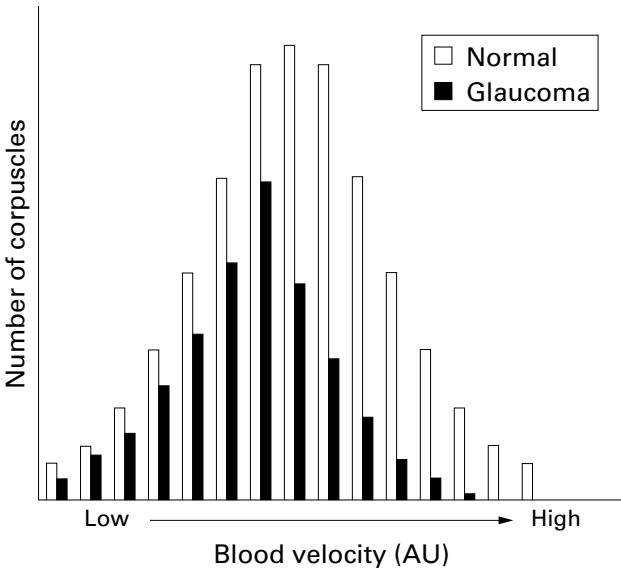

Figure 3 A proposed schematic model for reduced blood flow in glaucoma. The histogram shows a normal distribution of velocity values representing the total number of blood corpuscles moving in the retina over a period of time. The proposed model suggests that the mean velocity and number of cells is reduced in glaucoma with more profound loss in the higher velocity range.

either the retina or neuroretinal rim. Using the search strategy, glaucoma patients exhibited significantly lower blood flow, volume, and velocity in the neuroretinal rim when compared to age matched normal subjects; however, this difference was not replicated in the retina. These differences in blood flow were more marked when highest blood flow readings were used to compare between the two groups. For the lowest blood flow values, only the blood flow and velocity were significantly reduced in the diseased group.

The apparent variability in blood flow measures seen in any one image is largely due to the physiological pulsation of the retinal capillary bed which results in sizeable differences in blood flow measures with movement of the pixel frame by only one or two pixels. ${ }^{613}$ Analysis of ocular perfusion using the HRF technique and a standard $10 \times 10$ pixel grid samples data over a fixed area of interest and over a fixed period of time thereby sampling velocities in both the spatial and temporal domains. The flow of blood corpuscles will vary in terms of both the number and velocity of cells, providing a distribution of values of blood flow for the area. We therefore suggest that the sampling process yields a range of velocities with varying frequency, and that these distributions may differ with factors such as age ${ }^{14}$ or in the diseased eye compared to the normal eye. ${ }^{78}$ Figure 3 shows a schematic frequency histogram that illustrates a possible model for the total blood flow defect in glaucoma patients compared to normal subjects, with an overall reduction in both the mean velocity and number of moving corpuscles.

In this study, using the standard technique, no blood flow defects were identified, which may suggest that there were no deficiencies in the volume and rate of flow in our glaucoma sample, a finding that is at odds with most other reported literature. ${ }^{79}$ It is possible that this difference arises from the stage of glaucoma, which in our study was only mild to moderate based on visual field classifications, and may have been earlier than in previous studies. Alternatively there may be a sampling 
error such that blood flow differences were masked between the groups because the small sampling window does not allow for the inherent variations in flow within the local vascular neighbourhood. For example, some patients may have been assessed in an area of relatively high local flow while others were assessed in an area of low flow, thereby masking subtle differences between the two groups. In other words, a small measuring area, such as the $10 \times 10$ pixel window, may sample with a particular spatial bias resulting in particularly high or low velocity and flow. By using the search strategy, the local peaks and troughs of the velocity histogram can be compared between subject groups. The model of flow deficits in glaucoma that we propose is shown in Figure 3, and would suggest that reductions in flow in glaucoma patients are greater in the higher velocity range of the spectrum, with more subtle differences evident at lower velocities. This model is consistent with the absence in our data of a significant difference in the low values of blood velocity and the generally smaller absolute reductions and significance levels of blood flow, volume, and velocity found when the low flow values are used to compare between groups. With the high flow values, as can been seen from the graphs in Figure 2, the absolute level of change is much greater for all parameters. This model may not be applicable in other diseases or in glaucoma assessed at later stages of damage.

In a study by Chung et $a l^{\beta}$ two different methods of image analysis were employed, the first using a $10 \times 10$ pixel frame and the other in which the entire image was analysed to obtain blood flow measures. Findings using the standard $10 \times 10$ pixel window showed no difference between the glaucoma patients and normal subjects, a result which is in agreement with our own data. However, using their own pointwise analysis involving a larger sampling area, significant differences in blood flow measures existed between the two subject groups. These findings concur with our own observations that a small $(10 \times 10)$ pixel frame does not use a large enough sampling window to overcome the physiological variability in blood flow across the retina. While the pointwise analysis overcomes the local temporal and spatial variability in flow by using a larger window, this may not be appropriate in all investigations, particularly those in which smaller areas of the fundus are to be considered.

Previous studies have shown that blood velocity is lower in glaucoma patients when compared to age matched normal subjects in the retina, ${ }^{79}{ }^{15}$ optic nerve head, ${ }^{7}$ choroid, ${ }^{16}$ and ophthalmic artery. ${ }^{17}$ The results of our study offer some explanation for the discrepancy in findings obtained by different authors when determining blood flow deficits in glaucoma. For example, blood flow deficits have been reported in the retina, ${ }^{78}$ neuroretinal rim, ${ }^{7}$ and lamina cribrosa ${ }^{9}$ of glaucoma patients. In some studies blood flow appeared to be normal in the neuroretinal rim $^{9}{ }^{18}$ or peripapillary retina. ${ }^{19}$ Although the possibility of age effects may account for the absence of defects in some studies, ${ }^{19}$ a sampling error may offer a more reliable explanation.

The results from our study have implications for investigations in which blood flow in glaucoma patients and normal subjects are compared, or in longitudinal studies in which blood flow changes are monitored over time. In cross sectional studies, differences in blood flow between subject groups may not be identified due to inappropriate sampling of blood flow measures between individuals. In addition, when attempting to identify changes in blood flow measures in patients over time, as in longitudinal studies, alterations may not be detected because of the noise, resulting from the unintentional, randomised identification of high and low blood flow measures between visits. Our results suggest that the standard strategy resulted in a sampling error which is induced because too small an area of tissue has been evaluated without due account of local variations in velocity and the local tissue variations in flow mask real changes resulting from the disease process. The result of this was that no significant difference in blood flow parameters at the retina and neuroretinal rim were observed. If the local physiological variation in flow measures is not accounted for then false positive or false negative findings of change could be reported.

In summary, our data and the data of others ${ }^{8}$ suggest that blood flow deficits are more consistently and more readily identified when a larger sample area is considered, or when account is taken of the local physiological variation in blood flow. Our study suggests that this can be achieved using standard software by adopting a local search strategy and finding the lowest or highest values of blood flow, volume, and velocity to compare blood flow measures between subject groups.

1 Bonner RF, Nossal R. Principles of laser Doppler flowmetry. In: Shepherd AP, Oberg PA, eds. Laser Doppler blood flowmetry. Boston: Kluwer Academic Publishers, 1990:17-45. 2 Riva C, Harino S, Petrig B, et al. Laser Doppler flowmetry in the optic nerve. Exp Eye Res 1992;55:499-506.

3 Michelson G, Schmauss B. Two dimensional mapping of the perfusion of the retina and optic nerve head. $\mathrm{Br} \mathcal{F} O \mathrm{ph}$ thalmol 1995;79:1126-32.

4 Michelson G, Schmauss B, Langhans M, et al. Principle, validity, and reliability of the scanning laser Doppler flowmetry. F Glaucoma 1996;5:99-105.

5 Nicolela M, Hnik P, Schulzer M, et al. Reproducibility of retinal and optic nerve head blood flow measurements with (1997;6: 157-64.

6 Michelson G, Welsenbach J, Pal I, et al. Automated full field analysis of perfusion images gained by scanning laser Doppler flowmetry. Br f Ophthalmol 1998;82:1294-300.

7 Michelson G, Langhans M, Groh J. Perfusion of the juxtapapillary retina and the neuroretinal rim area in juxtapapillary retina and the neuroretinal rim area in

Primary open angle glaucoma. f Glaucoma 1996;5:91-8.
Chung H, Harris A, Kagemann L, et al. Peripapillary retinal Chung $\mathrm{H}$, Harris A, Kagemann L, et al. Peripapillary retinal
blood flow in Normal tension glaucoma. Br $\mathcal{F}$ Ophthalmol 1999;83:466-9.

9 Nicolela M, Hnik P, Drance S. Scanning laser Doppler flowmeter study of retinal and optic disk blood flow in glaucomatous patients. Am $\mathcal{F}$ Ophthalmol 1996;122:77583.

10 Kerr J, Nelson P, O'Brien C. A Comparison of ocular blood flow in untreated Primary open angle glaucoma and ocular hypertension. Am 7 Ophthalmol 1998;126:42-51.

11 Hoddapp E, Parrish RK, Anderson DR. Clinical decisions in glaucoma. St Louis: Mosby, 1993.

12 Bohdanecka Z, Orgul S, Prunte C, et al. Influence of acquisition parameters on hemodynamic measurements with the Heidelberg retinal flowmeter at the optic disc. F Glaucoma 1998;7:151-7.

13 Sullivan P, Cioffi G, Wang L, et al. The influence of ocular pulsatility on scanning laser flowmetry. Am $\mathcal{F}$ Ophthalmol 1999;128:81-7. 
14 Groh M, Michelson G, Langhans M, et al. Influence of age on retinal and optic nerve head circulation. Ophthalmology on retinal and op

15 Wolf S, Arend O, Sponsel WE, et al. Retinal hemodynamics using scanning laser ophthalmoscopy and hemorheology in chronic open-angle glaucoma. Ophthalmology 1993;100 1561-6.

16 Duijm HFA, VandenBerg TJTP, Greve EL. Choroidal haemodynamics in glaucoma. Br f Ophthalmol 1997;81: $735-42$.
17 Rojanapongpun P, Drance S, Morrison B. Ophthalmic artery flow velocity in glaucomatous and normal subjects. Br F Ophthalmol 1993;72:25-9.

18 Hollo G, Van den Berg T, Greve E. Scanning laser Doppler flowmetry in glaucoma. Int Ophthalmol 1997;20:63-70.

19 Hollo G, Greve E, VandenBerg T, et al. Evaluation of the peripapillary circulation in healthy and glaucoma eyes with scanning laser Doppler flowmetry. Int Ophthalmol 1997;20: scann $71-7$.

\section{Contributors please note:}

Communications from all countries except the UK and Republic of Ireland should be sent to Professor C Hoyt, Editor, British fournal of Ophthalmology, University of California, Department of Ophthalmology, 10 Kirkham Street, K 301, San Francisco, CA 94143-0730, USA (tel: 001415 502-6871; fax: 001415 514-1521).

Manuscripts from the UK and the Republic of Ireland should be sent to Professor Andrew Dick, UK Editor, British fournal of Ophthalmology, Division of Ophthalmology, University of Bristol, Lower Maudlin Street, Bristol BS1 2LX (tel: +44 (0) 0117 929-4496; fax: +44 (0) 117 929-4607). 